\title{
In situ assembly of the collagen-polyacrylamide interpenetrating network hydrogel: Enabling decoupled control of stiffness and degree of swelling
}

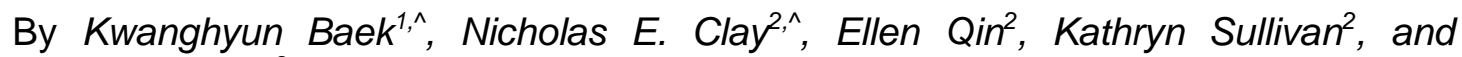
Hyunjoon Kong ${ }^{2, *}$

1. Department of Materials Science and Engineering, University of Illinois at UrbanaChampaign, Urbana, Illinois 61801, USA

2. Department of Chemical and Biomolecular Engineering, University of Illinois at Urbana-Champaign, Urbana, Illinois 61801, USA

\author{
* Corresponding author \\ $\wedge$ Signifies equal contribution \\ Department of Chemical and Biomolecular Engineering \\ Micro and Nanotechnology Laboratory \\ Institute of Genomic Biology \\ University of Illinois at Urbana-Champaign \\ Urbana, Illinois 61801, USA \\ E-mail: hjkong06@illinois.edu
}

\section{Abstract}

In the past several decades, hydrogels have been extensively studied for use in various biomedical and industrial applications. Successful use of hydrogels in these applications greatly relies on the ability to control mechanical and transport properties in a refined manner; however, current hydrogel design often encounters an undesirable inverse dependency between the degree of swelling and elastic modulus. To resolve this challenge, we hypothesized that an interpenetrating network (IPN) gel in which collagen fibers are chemically linked to a polymeric gel would allow us to control the elastic modulus over a broad range while reducing the change in the degree of swelling. We examined this hypothesis by preparing a collagen-polyacrylamide IPN gel via in situ assembly, so that two disparate networks are chemically linked via Michael reaction between collagen and polyacrylamide. The resulting IPN gel successfully demonstrated the independency between the degree of swelling and the elastic modulus. We suggest that the results of this study will be broadly useful in controlling hydrogel properties in an elaborate manner, in turn advancing various molecular and cell therapies.

Keywords: Interpenetrating network, Collagen, Polyacrylamide, Hydrogels, Elastic modulus, Degree of swelling 


\section{Introduction}

Hydrogels are formed from the chemical or physical cross-linking of polymers dissolved in aqueous media, and are increasingly used for various applications including sensing, actuation, controlled molecule delivery, and in vitro cell culture. [1-6] One of the important factors required for successful use of a hydrogel in these applications is the ability to control its mechanical stiffness over a broad range.[7] In particular, controlling gel stiffness helps maintain hydrogel structural integrity and regulates adhesion and phenotypic activities of adhered cells.[8] It is common to control stiffness by simply altering the number of cross-links in the gel; however, this approach often results in a significant change in gel pore diameter and permeability. Thus, increasing gel rigidity simultaneously decreases gel permeability and swelling ratio.[9]

To resolve this challenge, efforts were made to control mechanical properties of the gel with a minimal change in permeability. To this end, rigid nanofibers or nanoparticles were introduced into a hydrogel by mixing them with pre-gelled solution followed by activation of cross-linking reaction; however, few successful gels were reported, to our knowledge. Rather, these reinforcing components decreased the gel stiffness or ultimately inhibited gel formation.[10] These results were attributed to uncontrollable aggregation between nano-sized materials in a gel matrix as well as poor interfacial bonds with the gel-forming polymer.[11]

In this study, we hypothesized that the in situ assembly of interpenetrating polymeric network (IPN) gel consisting of polyacrylamide (PAAm) and collagen fibers would allow us to control gel stiffness over a broad range while reducing the change in gel permeability. Collagen fibers, a major 
extracellular component of various tissues in living organisms, present an elastic modulus and ultimate strength around 5 and $0.4 \mathrm{GPa}$, respectively. $[12,13]$ In particular, the in situ assembly process would facilitate a Michael reaction between the amine groups of collagen and the vinyl groups of acrylamide. Thus, the resulting collagen fibers are chemically connected to the PAAm gel without interfering with the cross-linking reaction between PAAm. We examined this hypothesis by systematically studying the effects of the concentration of collagen and the molar ratio between cross-linker and polyacrylamide on elastic modulus, swelling ratio, and the number of cross-links of the gel composite. In addition, we evaluated whether the resulting collagenhydrogel composite systems would regulate cellular adhesion behavior with their stiffness. We believe that the results of this study will greatly serve to improve the controllability of structure-property-functionality of various hydrogel systems.

\section{Experiments}

\subsection{Materials}

Acrylamide, N,N'-methylenebisacrylamide (bis-acrylamide), ammonium persulfate (APS), tetramethylethylenediamine (TEMED), sodium hydroxide $(\mathrm{NaOH})$, formaldehyde solution, trinitrobenzenesulfonic acid (TNBS, $5 \%(\mathrm{w} / \mathrm{w}))$, and $1 \mathrm{~N}$ hydrochloric acid $(\mathrm{HCl})$ were purchased from Sigma-Aldrich. Bovine collagen solution ( 3 or $6 \mathrm{mg} / \mathrm{mL}$ ) was purchased from Advanced Biomatrix.

Cell culture medium and its supplements, Dulbecco's modified Eagle's medium (DMEM), fetal bovine serum (FBS) and penicillin/streptomycin were purchased from Invitrogen. NIH 3T3 fibroblasts were purchased from American 
Type Culture Collection (ATCC). Phalloidin and 4',6-diamidino-2-phenylindole (DAPI) were purchased from Invitrogen.

2.2 In situ assembly and step fabrication of PAAm-collagen IPN gel

A $20 \%(w / w)$ acrylamide aqueous solution was prepared with different bis-acrylamide concentrations, including $0.03,0.1,0.3$, and $0.5 \%(w / w)$. The acrylamide/bis-acrylamide solution then was mixed with chilled collagen solution, either at $3 \mathrm{mg} / \mathrm{mL}$ or $6 \mathrm{mg} / \mathrm{mL}$. The volumetric ratio between the two solutions was kept constant at 1:1. The final concentration of the acrylamide was kept constant at $10 \%(\mathrm{w} / \mathrm{w})$ while the bis-acrylamide was varied from 0.015 to $0.25 \%(\mathrm{w} / \mathrm{w})$. Then, $1 \%(\mathrm{v} / \mathrm{v})$ of APS and $0.1 \%(\mathrm{v} / \mathrm{v})$ of TEMED were added to the gel solution to activate cross-linking. These gels were known as the "IPN" gels or the "in situ fabrication" gels. For control experiments, collagen-free polyacrylamide gels were prepared with the same masses of acrylamide and bis-acrylamide. To form a gel, the pre-gelled solution was placed between two glass plates with a 1-mm thick spacer. Afterwards, hydrogel disks with $10 \mathrm{~cm}$ diameter and $1 \mathrm{~mm}$-thickness were punched out using a biopsy punch with a $10 \mathrm{~cm}$-diameter.

Separately, a pure collagen gel was prepared by changing $\mathrm{pH}$ of the collagen stock solution to $7.0-7.4$ by adding $1 \mathrm{M} \mathrm{NaOH}$. Then, the mixture was incubated at $37^{\circ} \mathrm{C}$ for 3 hours to form a collagen gel. The pre-formed collagen gel was mixed with the acrylamide/bis-acrylamide solution by a vortex mixer. This gel was termed the "step fabrication" or "step" gel. The final concentration of the acrylamide was kept constant at $10 \%(\mathrm{w} / \mathrm{w})$ while the bis- 
acrylamide was varied from 0.015 to $0.25 \%(w / w)$. Finally, the mixture was mixed with APS and TEMED to activate gel formation.

2.3 Measurements of elastic moduli and swelling ratios of hydrogels

Following incubation of hydrogel disks in DI water for at least $24 \mathrm{~h}$ at room temperature, the disks with $10 \mathrm{~cm}$-diameter and $1 \mathrm{~mm}$-thickness were uniaxially compressed at a rate of $1 \mathrm{~mm} / \mathrm{min}$ using a mechanical testing system (MTS Systems Insight). The tester measured the force required to keep the deformation rate of the gel constant. Then, the force was automatically divided by cross-sectional area of the gel, in order to calculate the stress. In parallel, strain was calculated by dividing the magnitude of gel thickness change by the original thickness of the gel. Finally, elastic modulus was calculated from the slope of a stress vs. strain curve at the first $10 \%$ strain. This parameter is termed "compressive elastic modulus" or simply "elastic modulus".

In parallel, the swelling ratio was characterized by measuring the mass of water in each gel. First, the mass of the hydrated gel was measured after incubation in DI water over 24 hours at room temperature. Then, the gel was lyophilized to measure the dried solid mass. Finally, the degree of swelling $(Q)$ was calculated from a mass ratio of the hydrated gel to the dried solid, $Q_{m}$, using the Eq. (1):

$$
Q=v_{2}^{-1}=\rho_{P}\left(\frac{Q_{m}}{\rho_{S}}+\frac{1}{\rho_{P}}\right)
$$

where $\rho$ s was the density of water $\left(1 \mathrm{~g} / \mathrm{cm}^{3}\right)$ and $\rho p$ was the density of PAAm $\left(1.13 \mathrm{~g} / \mathrm{cm}^{3}\right)$. 


\subsection{Tensile and rheological testing of hydrogels}

All tensile tests were performed in air and at room temperature, using a mechanical testing system (Insight, MTS) with a 250-N load cell. The rate of stretch was kept constant at $2 \mathrm{~mm} / \mathrm{min}$, and the gel specimens were cut into 30 x $10 \times 1 \mathrm{~mm}$ pieces and were clamped so that the initial length was kept constant at $5 \mathrm{~mm}$, width at $10 \mathrm{~mm}$, and thickness at $1 \mathrm{~mm}$. We then defined tensile elastic modulus, maximum strength and fracture strain as follows: tensile elastic modulus was calculated from the slope of the linear section of the stress-strain curve at the first $10 \%$ strain; maximum strength was defined as the maximum stress at the fracture strain; and fracture strain was defined as the strain at which the gel sample fractures.

To measure shear storage modulus, gel-forming solutions were placed on a parallel plate configuration on a rheometer (DHR-3, TA instruments). After gelling for 15 min, a frequency sweep measurement was conducted within a linear elastic region determined by the amplitude sweep test. For a given gel condition, shear storage modulus was defined as the storage modulus value at $1 \mathrm{~Hz}, 0.1 \%$ strain for a frequency sweep.

\subsection{Microscopic analysis of hydrogel structure}

After fabrication, hydrogels were flash-frozen with liquid nitrogen. Then, the frozen gel was fractured, followed by lyophilization. Dried samples were cut by a razor and attached on a sample holder using carbon tape. The samples were coated with gold/platinum for charge dissipation. The fractured surface of the freeze-dried hydrogel samples was imaged using a scanning electron 
microscopy (Hitachi S4800). The acceleration voltage was tuned to $15 \mathrm{kV}$ and the emission current was kept constant at $10 \mu \mathrm{A}$.

2.6 Analysis of chemical bonds between polyacrylamide and collagen

The 2,4,6-trinitrobenzene sulfonate (TNBS) assay was used to measure the relative concentration of primary amines in each hydrogel. Briefly, $25 \mu \mathrm{L}$ of TNBS was diluted in $5 \mathrm{~mL}$ of phosphate buffered saline solution (PBS). Then, volumes of each hydrogel (about $800 \mu \mathrm{L}$ ) were incubated in $0.5 \mathrm{~mL}$ of the diluted TNBS solution for $18 \mathrm{~h}$ at $37^{\circ} \mathrm{C}$. Afterwards, $100 \mu \mathrm{L}$ of TNBS solution was drawn off from each sample. Then, $50 \mu \mathrm{L}$ of $1 \mathrm{~N} \mathrm{HCl}$ was added to each $100 \mu \mathrm{L}$ of TNBS solution to quench the assay. The absorbance of the quenched TNBS solution was read at a wavelength of $335 \mathrm{~nm}$ (Tecan Infinite F200Pro).

To monitor the progress of the Michael reaction, a dilute pre-gel solution with $10 \mathrm{mg} / \mathrm{mL}$ acrylamide, $0.25 \mathrm{mg} / \mathrm{mL}$ bis-acrylamide, and $0.3 \mathrm{mg} / \mathrm{mL}$ collagen was prepared. After thorough mixing, $200 \mu \mathrm{L}$ aliquots were prepared. At 5, 10, 20 and 30 min, a concentrated amount of TNBS $(200 \mu \mathrm{L}$ of $\sim 0.37 \%$ (w/w)) was added to a separate aliquot. Two minutes after adding the TNBS solution, the TNBS reaction was quickly quenched by adding $100 \mu \mathrm{L}$ of $1 \mathrm{~N} \mathrm{HCl}$. Afterwards, the absorbance of the quenched TNBS solution was read at a wavelength of $335 \mathrm{~nm}$, and then normalized to the initial time point (5 minutes).

\subsection{Analysis of cell adhesion on hydrogels}

Sterile PAAm-collagen IPN hydrogels with different elastic moduli were prepared by first filtering the pre-gel solution through a $0.22 \mu \mathrm{m}$ filter and then activating the cross-linking reaction. Then, $1.0 \times 10^{4} \mathrm{NIH} 3 \mathrm{~T} 3$ fibroblasts 
suspended in $100 \mu \mathrm{L}$ of DMEM were plated on top of each hydrogel. The celladhered gel disks were incubated in DMEM supplemented with $10 \%$ (v/v) FBS and $1 \%(\mathrm{v} / \mathrm{v})$ penicillin/streptomycin at $37^{\circ} \mathrm{C}$. After 4 hours, the hydrogel was washed with PBS to remove unbound cells and further incubated in fresh DMEM. After 24 hours, cells were fixed with $4 \%$ formaldehyde solution. To visualize the actin filaments and nuclei of the cells adhered on the hydrogel, the cells were incubated with Alexa Fluor 488 phalloidin and DAPI following permeabilization. The stained cells on the hydrogel were then imaged using a laser-scanning confocal microscope (Zeiss LSM 740). The cell area (i.e., the area that was positive for actin staining) was measured using $\mathrm{NIH}$ ImageJ software. The cell area of at least 10 cells was measured, taken from multiple images per condition.

\subsection{Statistical analysis}

At a minimum, three repeats were done for each experimental group. Statistical significance was determined via Student's t test (for two groups) or a one-way ANOVA with post-hoc Tukey's (for more than two groups). Significance was defined as $p<0.05$. The data is presented with mean \pm standard error of the mean from the three samples per each condition unless indicated otherwise. 
3. Results and discussion

3.1 In situ assembly of PAAm-collagen IPN gel and analysis of mechanical properties

Mixing acrylamide/bis-acrylamide solution with chilled, acidic collagen stock solution led to a transparent solution with no aggregation between collagen molecules. Adding APS and TEMED to the pre-gelled solution activated gel formation. The cross-linking density of PAAm in both IPN gels and pure PAAm gels was modulated by changing the molar ratio between bisacrylamide (i.e., cross-linker) and acrylamide, termed as $M$ bis.

The resulting PAAm-collagen IPN gels as well as the pure PAAm gel were uniaxially compressed to evaluate the combined effects of collagen and Mbis on stiffness. At a given collagen concentration of $0.15 \%(w / w)$, the compressive elastic moduli of PAAm-collagen IPN gels were higher than that of the pure PAAm gel. Interestingly, the difference in elastic moduli between the IPN gels and the pure PAAm gels became larger with increasing Mbis (Figure 1a \& 1b). Therefore, at $M_{\mathrm{bis}}$ of $2.3 \%$, the average elastic modulus of the IPN gel was three-fold higher than that of the pure PAAm gel. As such, the IPN gel system allowed us to control the elastic modulus over a broader range (i.e., from 12 to $32 \mathrm{kPa}$ ) than the pure PAAm gel (i.e., 2 to $10 \mathrm{kPa}$ ). Note that pure collagen hydrogels alone have a storage modulus of only around 10-100 Pa. [14] 
(a)

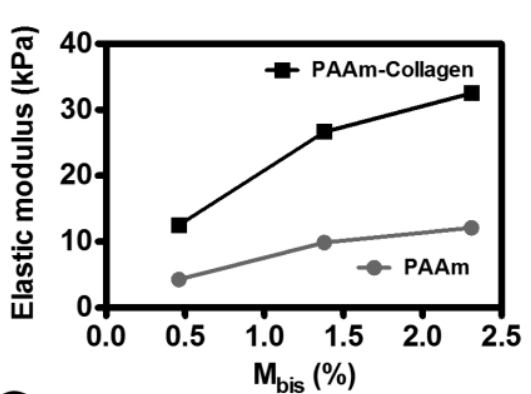

(c) $\pi$

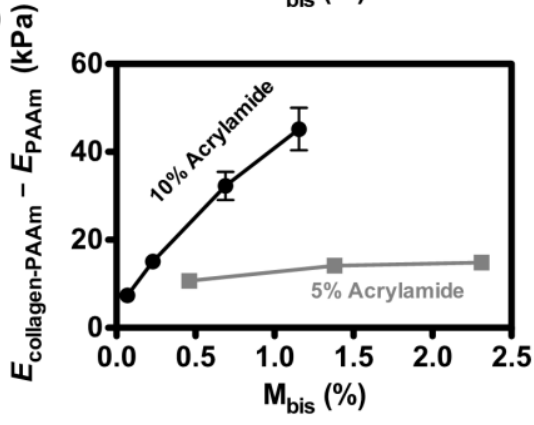

(b)

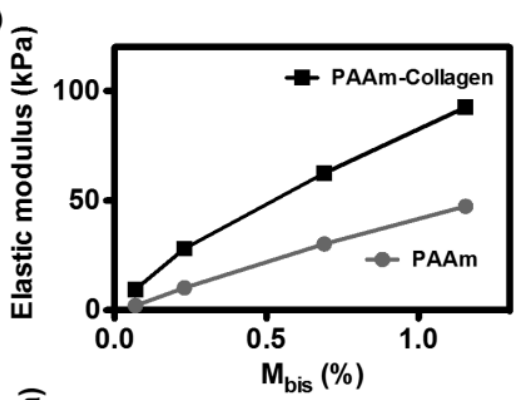

(d)

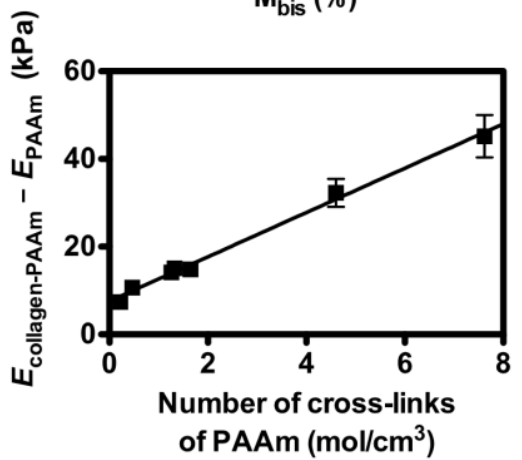

Figure 1. Mechanical properties of the pure PAAm hydrogel (noted as PAAm) and collagen-PAAm IPN hydrogels (noted as collagen-PAAm). (a) Effects of the molar ratio between bis-acrylamide and acrylamide $\left(M_{\text {bis }}\right)$ on elastic moduli of hydrogels at an acrylamide concentration of $5 \%(w / w)$. (b) Effects of $M_{\text {bis }}$ on elastic moduli of hydrogels at an acrylamide concentration of $10 \%(w / w)$. (c) Effects of $M_{\text {bis }}$ on the difference of elastic modulus between the pure PAAm and the collagen-PAAM IPN gels ( $\left.E_{\text {collagen-PAAm }}-E_{P A A m}\right)$. (d) A universal relationship between $E_{\text {collagen-PAAm }}-E_{\text {PAAm }}$ and the number of cross-links of PAAm.

Increasing acrylamide concentration in the IPN gel further broadened the range of elastic modulus to be controlled. Specifically, at PAAm concentration of $10 \%(\mathrm{w} / \mathrm{w})$, the elastic modulus of the IGN gel could be varied from 10 to $93 \mathrm{kPa}$ by altering $M$ bis from 0.1 to $1.2 \%$ (Figure $1 \mathrm{~b}$ ). In contrast, increasing $M_{\text {bis }}$ from 0.1 to $1.2 \%$ with the pure PAAm gel led to an increase of the elastic modulus only from 2 to $47 \mathrm{kPa}$. The difference of the elastic modulus between the IPN gel and the pure PAAm gel $(\Delta E)$ was linearly related to $M$ bis at both PAAm concentration of 5 and $10 \%(w / w)$ (Figure 1c).

In addition, the number of cross-links of the gel $(N)$ was calculated with the measured elastic modulus and the degree of swelling, using an elastic network model (Eq. (2)): 


$$
N=\frac{k Q^{-1 / 3}}{R T}
$$

where $k$ is the shear modulus, $Q$ is the swelling ratio, $R$ is the gas constant (8.314 $\left.\mathrm{Jmol}^{-1} \mathrm{~K}^{-1}\right)$ and $\mathrm{T}$ is the temperature at which the elastic modulus was measured (Figure 1d). Note that $\mathrm{k}$ is calculated from the slope of compressive elastic modulus versus $-\left(\lambda-\lambda^{-2}\right)$, whereby $\lambda$ is strain. $[15,16]$

Interestingly, the linear dependency of $\Delta E$ on $N$ fell into a universal curve, regardless of the PAAm concentration of the gel (Figure 1d). This result clearly states that the linear dependency of $\Delta E$ on $M$ bis at varied PAAm concentrations is attributed to the increase of the number of cross-links between PAAm molecules.

3.2 Effects of the assembly process on microstructure and properties of IPN hydrogels

The effects of the hydrogel assembly methods on the resulting microstructure and stiffness were further examined to highlight the advantages of in situ IPN gel assembly. A control gel, which was termed as the "step fabrication" gel, was prepared by first mixing pre-formed collagen gels with PAAm/bis-acrylamide solutions and subsequently activating the radical crosslinking reaction between PAAm.

According to SEM images of the freeze-dried PAAm-collagen hydrogels, gels prepared via the in situ fabrication and those prepared via the step fabrication displayed the presence of collagen fibers associated with the PAAm matrix (Figure 2a). The average diameter of the collagen fiber was approximately $60 \mathrm{~nm}$, which was similar to the collagen fibers formed in the pure collagen gel. However, the collagen fibers were more uniformly distributed 
in the IPN gel prepared by the in situ fabrication. In contrast, the gel prepared via the step fabrication exhibited heterogeneous distribution of collagen fibers. Additionally, some of the collagen fibers were aggregated in the PAAm matrix.

(a)
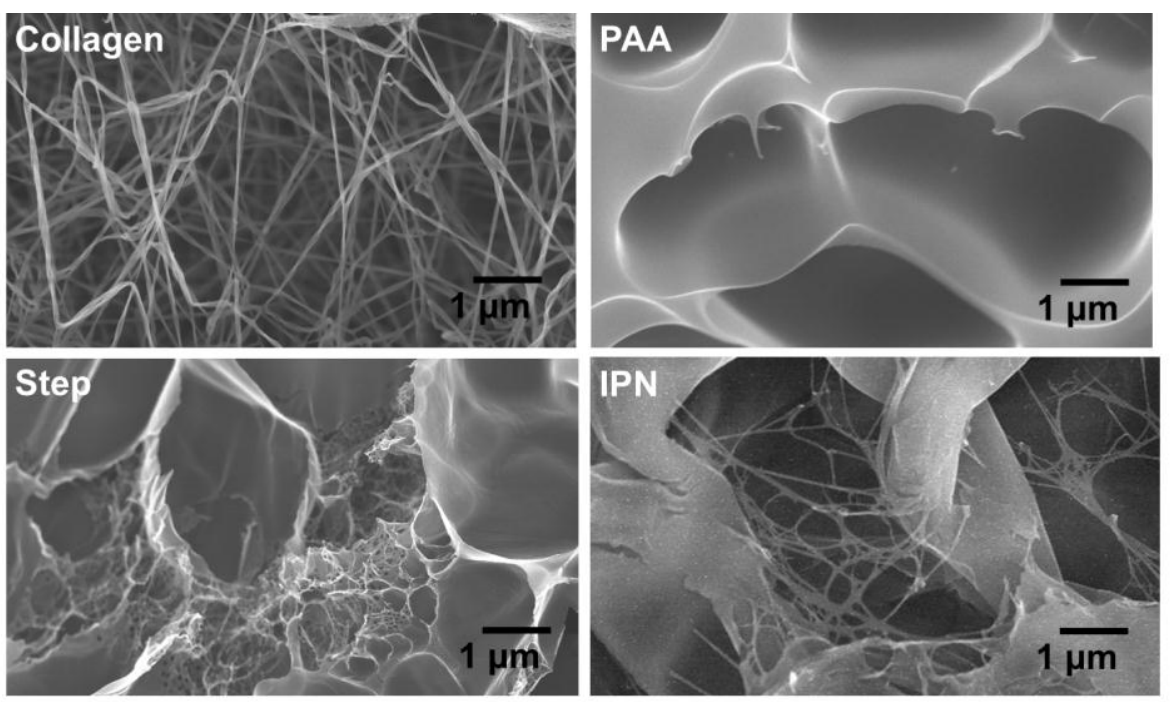

(b)

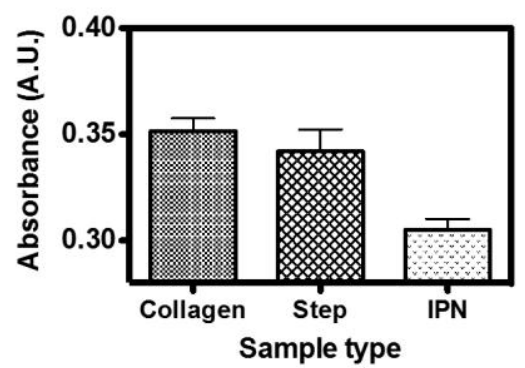

Figure 2. Microstructural analysis of hydrogels prepared with different composition and process. (a) Scanning electron microscope (SEM) images of the cross-section of the collagen gel, the pure PAAm gel, the collagen-PAAm gel prepared via the step fabrication process, and the collagen-PAAm IPN gel prepared via the in situ fabrication. (b) TNBS assay to quantify the number of unreacted primary amine groups of collagen. Absorbance at the wavelength of $335 \mathrm{~nm}$ was used as an indicator of the primary amine group.

A TNBS assay was performed to examine effects of the gel assembly on chemical connectivity between collagen and PAAm. Note that TNBS reacts with primary amine groups to produce chromogenic derivatives with UV absorbance at $335 \mathrm{~nm}$. Using this assay, the relative number of unreacted primary amine groups of the collagen in each gel can be quantified (Figure 2b). No difference 
in absorbance between the pure collagen gel and the control gel prepared by the step fabrication was noted. However, the IPN gel prepared by the in situ fabrication displayed a significantly lower absorbance value than the two other conditions, which suggests that the IPN gel has fewer primary amines. This result confirms the Michael reaction between collagen and acrylamide during in situ fabrication, thus leading to the chemical connection between collagen $\begin{array}{llll}\text { fibers and } \text { PAAm } & \text { (Scheme }\end{array}$

\section{a) In Situ Fabrication}
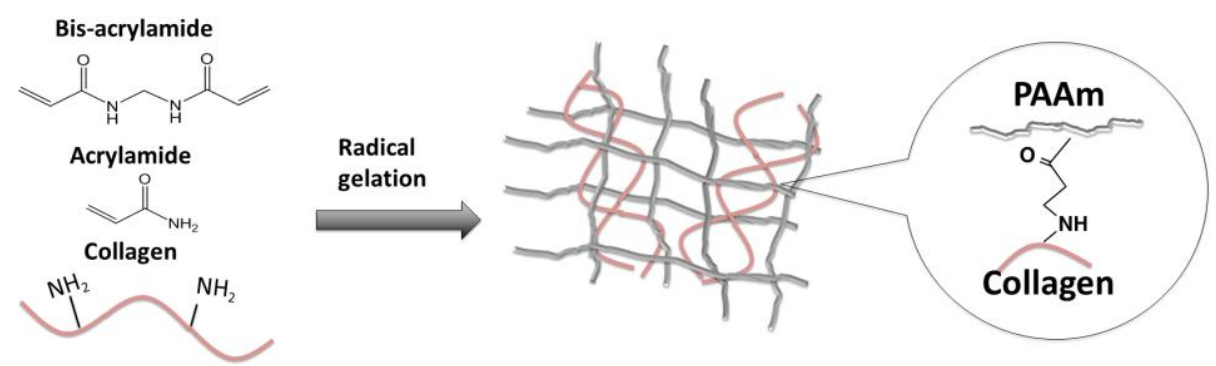

b) Step Fabrication
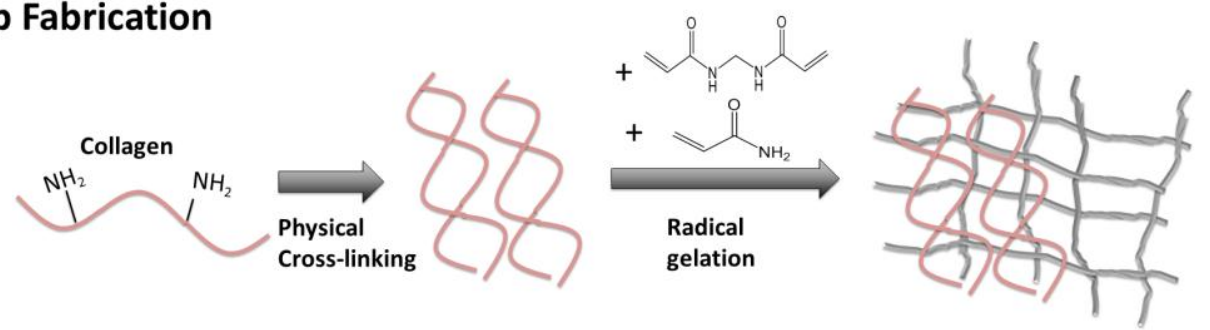

Scheme 1. Microstructure formed with in situ gel fabrication and step fabrication.

Schematics describing microstructure of (a) collagen-PAAm gel prepared by the step fabrication and (b) collagen-PAAm IPN gel prepared by the in situ fabrication.

According to the TNBS assay conducted with a dilute pre-gel mixture, a significant number of amine groups was consumed about 10 minutes after the reaction starts (Figure S1). Based on the gelation kinetics of collagen-free acrylamide, we propose that that radical polymerization and the Michael addition reaction likely happen simultaneously. 
Thiol groups on collagen, which can potentially react with vinyl groups of acrylamide, made minimal roles in mechanical properties of the gel, according to control experiments we conducted with dithiothreitol (DTT), a model divalent thiol compound. The gel formed from a mixture of acrylamide and DTT did not present a significant difference in storage modulus compared to the PAAm gel formed without DTT (data not shown). Therefore, the most likely mechanism for IPN gel fabrication involved the reaction of primary amines from collagen with the vinyl bonds of acrylamide.

The difference of microstructure in these two gel systems significantly influenced elastic moduli and number of cross-links (Figure 3a). The elastic modulus of the gel prepared by the step fabrication was not significantly different from that of the pure PAAm gel. In contrast, the IPN gel prepared by the in situ fabrication presented two-fold higher elastic modulus than two other conditions (i.e., the pure PAAm gel and the gel prepared by the step fabrication). Using Eq. 2, the number of cross-links for the in situ fabricated gel was two times greater than the two other conditions (Figure 3b).

(a)

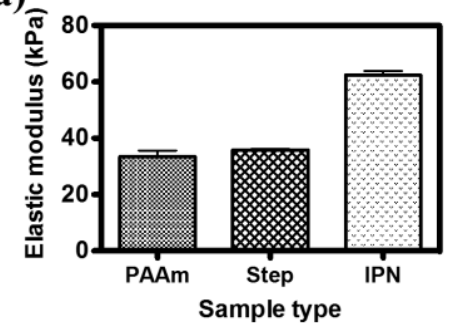

(b)

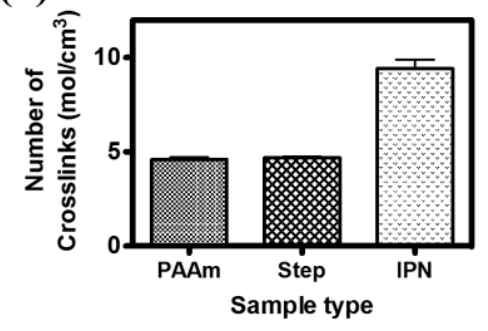

Figure 3 Analysis of (a) the elastic modulus and (b) the number of cross-links prepared with different composition and. 'PAAm' represents the pure PAAm gel. "step" represents the collagen-PAAm gel prepared via the step fabrication process. "IPN" represents the collagen-PAAm IPN gel prepared via in situ fabrication process. Concentrations of acrylamide, bis-acrylamide and collagen were kept 10, 0.15 and $0.15 \%(w / w)$, respectively, for all conditions. 
3.3 Decoupling of elastic modulus and swelling ratio attained by the in situ fabrication of IPN gel

Incorporation of collagen into the PAAm gel via in situ assembly substantially reduced the inverse dependency between elastic modulus and the degree of swelling. Increasing $M_{\text {bis }}$ from 0.1 to $1.2 \%$ led to a significant decrease of swelling while increasing elastic modulus from 2 to $47 \mathrm{kPa}$ (Figure 4a-1). However, incorporation of collagen interestingly broadened the range of elastic modulus to be controlled while limiting the decrease of swelling (Figure 4a-2\& 4a-3). Therefore, the collagen-PAAm gel prepared with $0.3 \%(w / w)$ collagen via in situ assembly exhibited a minimal change in the degree of swelling despite a large increase of the compressive elastic modulus from 15 to $90 \mathrm{kPa}$ (Figure 4b). With the addition of collagen, the tensile properties of PAAm hydrogels similarly increased (Table S1). The addition of collagen at 0.15 and $0.3 \%(\mathrm{w} / \mathrm{w})$ resulted in gels with higher tensile elastic moduli and maximum strength than the controls without collagen. Similarly, shear storage modulus, as determined from rheological testing, increased with increasing collagen and $M$ bis (Table S2).

The degree of dependency $(k)$ was further quantified by fitting the curves of the degree of swelling versus compressive elastic modulus to first-order exponential decay (Eq. (3)),

$$
Y=\left(Y_{0}-Y_{L}\right) e^{-k x}+Y_{L}
$$

whereby $Y_{0}$ and $Y_{L}$ are the infinite degrees of swelling and zero degree of swelling, respectively. [15] 
Interestingly, $\mathrm{k}$ decreased with increasing collagen concentration (Figure 4c). The collagen-induced decoupling of elastic modulus and the degree of swelling were not observed with the gel prepared via the two-step process (data not shown).
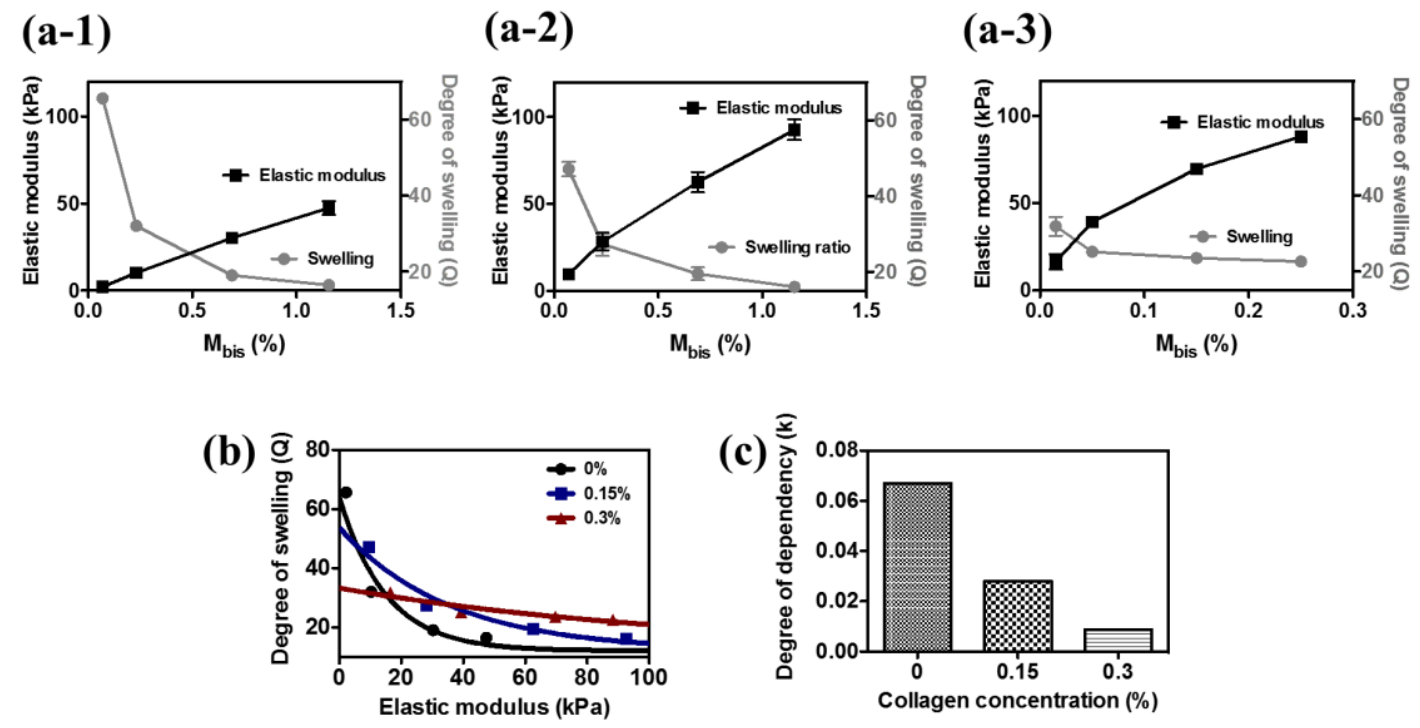

Figure 4. Effects of collagen on decoupling the inverse dependency between the degree of swelling and the elastic modulus of hydrogels. (a) Effects of $M_{\text {bis }}$ on the elastic modulus and the degree of swelling for (a-1) pure PAAm gel, (a-2) collagenPAAm IPN gel with collagen concentration of $0.15 \%$, and (a-3) collagen-PAAm IPN gel with collagen concentration of $0.30 \%$. (b) Effects of collagen concentration on the inverse dependency between the degree of swelling and the elastic modulus. (c) The degree of inverse dependency altered with the collagen concentration. The degree of inverse dependency was quantified by fitting curves in (b) to the first-order exponential decay. Concentrations of acrylamide, bis-acrylamide were kept 10 and $0.15 \%(w / w)$, respectively.

\subsection{Cell adhesion modulated by stiffness of collagen-PAAm IPN gel}

Finally, the resulting collagen-PAAm IPN gel was used as a substrate to examine the effects of material stiffness on cell adhesion. Cells did not adhere to the bare PAAm gel while they actively adhere to the IPN gel. Cells were maximally extended on the gels with compressive elastic moduli of 30 and 60 $\mathrm{kPa}$ ( $\mathrm{Mbis}$ of 0.23 and $0.69 \%$ ), according to quantification made with fluorescent 
cell images (Figure 5). Decreasing compressive elastic modulus to $10 \mathrm{kPa}$ (Mbis of $0.07 \%$ ) substantially reduced the extent of cell stretching.

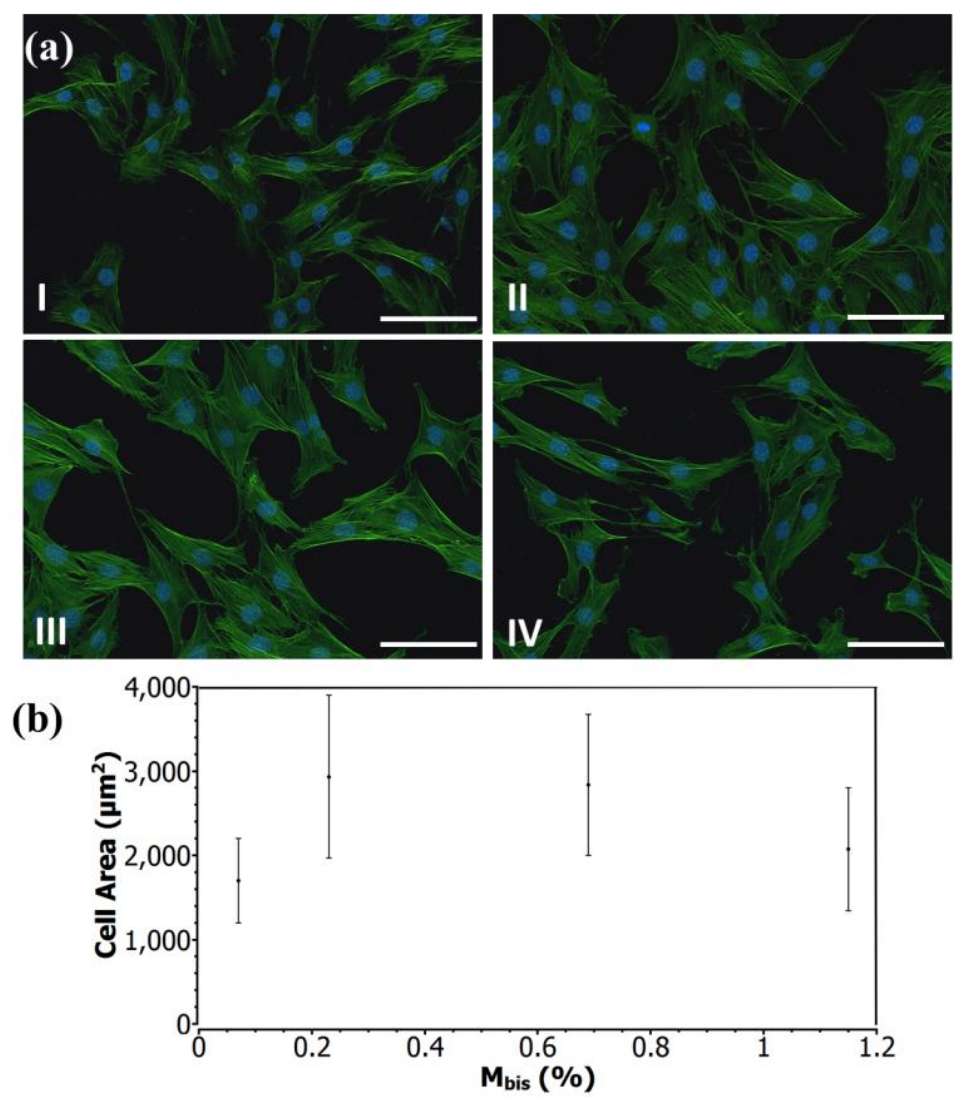

Figure 5. Demonstration of the capability of the IPN gel to modulate cellular spreading with its elastic modulus (a) Confocal microscope images of NIH 3T3 fibroblasts adhered to the collagen-PAAm. I, II, III and IV represent collagen-PAAm hydrogels prepared at $M_{\text {bis }}$ of $0.07,0.23,0.69$ and $1.15 \%$, respectively. Concentrations of acrylamide and collagen were kept $10 \%$ and $0.15 \%$, respectively for all conditions. Scale bars are $100 \mu \mathrm{m}$. (b) Effects of $M_{\text {bis }}$ on cell area on the IPN gel. Data points and error bars in each plot represent mean values and standard deviations from at least 10 cells per condition, respectively.

\section{Discussion}

In summary, our results demonstrate a new method to assemble a celladherent hydrogel system which presents a broad range of elastic modulus while minimizing changes in the degree of swelling. The in situ cross-linking reaction of PAAm mixed with collagen enabled us to change compressive elastic modulus from 10 to $100 \mathrm{kPa}$ by altering the concentration of bis- 
acrylamide, a cross-linker. In contrast, increasing bis-acrylamide concentration of the pure PAAm gel altered a smaller range of the elastic modulus while making a bigger variation in the degree of swelling. Based on preliminary cell studies, the resulting collagen-PAAm IPN gel served as a platform on which cells could readily adhere.

Our results showed collagen made a more significant influence on stiffening the gel at a higher bis-acrylamide (i.e., cross-linker of PAAm) concentration. We suggest that this integrated effect of collagen and bisacrylamide concentration on increasing compressive elastic modulus is partially attributed to the intrinsic stiffness of collagen fibers formed in the gel matrix. It is well agreed that a single collagen fiber formed from physical association of collagen fibrils presents an elastic modulus of up to 5 GPa. [12,13] As characterized with the TNBS assay, we interpret that the in situ assembly of IPN gels enables these stiff collagen fibers to chemically bond to bis-acrylamide as well as PAAm, such that the collagen-induced stiffening effect becomes more significant with increasing bis-acrylamide concentration. Previous results show that the chemical bonding between two separate, cross-linked networks in the IPN synergistically increases rigidity of the IPN. $[17,18]$ In addition, the uniform distribution of the stiff collagen fibers in the IPN gel compared with the step fabrication gel with inhomogeneous collagen distribution should be another factor to increase gel stiffness.

Another highlight of this study was the finding that the IPN gel decouples the inverse dependency between the degree of swelling and the elastic modulus of the gel. Therefore, the more rigid collagen-PAAm IPN gel could present a higher degree of swelling than the softer, pure PAAm gel. It is 
understood that increasing the cross-linking density of a polymeric gel increases the hydrophobicity of a matrix, thus limiting entry of water into the gel. We suggest that the collagen fibrous networks chemically linked with the PAAm network facilitate water entry into the gel even with the larger number of crosslinks between PAAm. This interpretation is supported by the finding that increasing collagen concentration reduces the degree of dependency between the degree of swelling and elastic modulus. In addition, the step fabrication gel in which the collagen network is not chemically bound to the PAAm gel exhibited a clear inverse dependency between the degree of swelling and elastic modulus. This result also implicates the important role of chemical bonds between collagen and PAAm in decoupling stiffness and swelling ratio.

The resulting IPN gel presents several advantages for use as an in vitro cell culture platform. For instance, PAAm gels immobilized with cell adhesion proteins including collagen have been used to culture cells of interest on their surfaces. It is common to chemically conjugate cell adhesion moieties using a linker such as sulfosuccinimidyl 6-(4'-azido-2'-nitrophenylamino)hexanoate (sulfo-SANPAH) and acrylate-PEG-N-hydroxysuccinimide (Acry-PEG-NHS), which require laborious, costly multi-conjugation and purification steps.[19] In contrast, this in situ IPN gel assembly will enable one to assemble the cell adherent gel with desired mechanical properties via a simple, single step. In this study, we demonstrate that cell area is largely constant despite the considerable changes in gel stiffness (most notably at Mbis of $0.23 \%$ and $0.69 \%$, despite the two-fold increase in elastic modulus). This result suggests that collagen density has a stronger effect on cell adhesion than hydrogel stiffness. For future work, we will spatially modulate surface density of collagen on the 
collagen-PAAm gel, in order to examine the combined effects of collagen density and stiffness on cell phenotype.

Additionally, with the decoupled control of the degree of swelling and the elastic modulus advantages, the IPN gel will be able to enhance quality of drug or cell delivery and tissue regeneration. For instance, a gel loaded with cells or drug molecules should be able to retain its structural integrity and also be permeable to facilitate biomolecular transports. However, the inverse dependency between permeability and stiffness found in a conventional gel system often exhibit limited molecular release and cell viability in a rigid matrix. The collagen-PAAm IPN gel system will greatly resolve such challenges, so as to elaborate molecular release and cell activities in a 3D gel matrix.

While there have been several studies to design IPN gels prepared from either collagen or acrylamide [20,21], this study reports, to the best of our knowledge, the capability of the collagen gel to independently control mechanical properties of the synthetic gel for the first time. In addition, the IPN gel system reported herein present the unique ability to tune the inverse dependency between swelling ratio and elastic modulus of the gel system.

\section{Conclusion}

In conclusion, this study demonstrated a simple but advanced method to control the elastic modulus and the degree of swelling of a cell adherent IPN hydrogel. The capability of the IPN gel to control elastic modulus over a broader range than control conditions was attributed to the covalent chemical bonds between collagen fibers and PAAm. This decoupled control of the degree of swelling and elastic modulus was likely because collagen networks linked to 
the polymeric network limit the cross-linking-induced increase of the hydrophobic environment. The resulting IPN gel could enable cellular adhesion as well. Overall, the gel assembly developed in this study will be broadly applicable to a wide array of gel systems formed from radical cross-linking reaction between acrylate or methacrylate groups. Additionally, the results of this study will serve to better understand effects of extracellular microenvironment and also improve the quality of various molecular and cell therapies.

Acknowledgement

This work was supported by the National Science Foundation (CBET1340491 to H.K., STC-EBICS Grant-0939511 to H.K. and K.S., and IGERTCMMB Grant 0965918 to E.Q.) and Korea Institute of Industrial Technology (JE 140004 to H.K.). N.C. acknowledges support from the Dow Chemical Company Fellowship.

References

[1] J.H. Holtz, S.A. Asher, Polymerized colloidal crystal hydrogel films as intelligent chemical sensing materials, Nature. 389 (1997) 829-832. doi:10.1038/39834.

[2] D. Buenger, F. Topuz, J. Groll, Hydrogels in sensing applications, Prog. Polym. Sci. 37 (2012) 1678-1719.

[3] B.P. Lee, S. Konst, Novel Hydrogel Actuator Inspired by Reversible Mussel Adhesive Protein Chemistry, Adv. Mater. 26 (2014) 3415-3419. doi:10.1002/adma.201306137.

[4] K. Baek, J.H. Jeong, A. Shkumatov, R. Bashir, H. Kong, In Situ SelfFolding Assembly of a Multi-Walled Hydrogel Tube for Uniaxial Sustained Molecular Release, Adv. Mater. 25 (2013) 5568-5573. doi:10.1002/adma.201300951.

[5] A.S. Hoffman, Hydrogels for biomedical applications, Adv. Drug Deliv. Rev. 64 (2012) 18-23. doi:10.1016/j.addr.2012.09.010.

[6] J.R. Tse, A.J. Engler, Current Protocols in Cell Biology, John Wiley \& Sons, Inc, Hoboken, NJ, USA, 2001. doi:10.1002/0471143030.cb1016s47.

[7] K. Saha, A.J. Keung, E.F. Irwin, Y. Li, L. Little, D.V. Schaffer, et al., 
Substrate Modulus Directs Neural Stem Cell Behavior, Biophys. J. 95 (2008) 4426-4438. doi:10.1529/biophysj.108.132217.

[8] J.J. Schmidt, J. Rowley, H.J. Kong, Hydrogels used for cell-based drug delivery, J. Biomed. Mater. Res. 87A (2008) 1113-1122. doi:10.1002/jbm.a.32287.

[9] K.H. Bae, L.-S. Wang, M. Kurisawa, Injectable biodegradable hydrogels: progress and challenges, J. Mater. Chem. B. 1 (2013) 5371. doi:10.1039/c3tb20940g.

[10] C. Cha, S.Y. Kim, L. Cao, H. Kong, Decoupled control of stiffness and permeability with a cell-encapsulating poly(ethylene glycol) dimethacrylate hydrogel, Biomaterials. 31 (2010) 4864-4871. doi:10.1016/j.biomaterials.2010.02.059.

[11] P. Thoniyot, M.J. Tan, A.A. Karim, D.J. Young, X.J. Loh, NanoparticleHydrogel Composites: Concept, Design, and Applications of These Promising, Multi-Functional Materials, Adv. Sci. (2015). doi:10.1002/advs.201400010.

[12] M.P.E. Wenger, L. Bozec, M.A. Horton, P. Mesquida, Mechanical Properties of Collagen Fibrils, Biophys. J. 93 (2007) 1255-1263. doi:10.1529/biophysj.106.103192.

[13] M. Minary-Jolandan, M.-F. Yu, Nanoscale characterization of isolated individual type I collagen fibrils: polarization and piezoelectricity, Nanotechnology. $20 \quad$ (2009) 085706. doi:10.1088/09574484/20/8/085706.

[14] Y. Liang, H. Kong, Y. Tong. Generation of cell-instructive collagen gels through thermodynamic control. ACS Macro Letters. 2 (2013) 10771081. dx.doi.org/10.1021/mz400512.

[15] K.S. Anseth, C.N. Bowman, L. Brannon-Peppas, Mechanical properties of hydrogels and their experimental determination, Biomaterials. 17 (1996) 1647-1657. doi:10.1002/adma.200904179.

[16] C. Cha, R. H. Kohman, H. Kong. Biodegradable polymer crosslinker: independent control of stiffness, toughness, and hydrogel degradation rate. Adv. Fun. Mater. 19 (2009) 3056-3062. doi: 10.1002/adfm.200900865.

[17] Y.-H. Na, T. Kurokawa, Y. Katsuyama, H. Tsukeshiba, J.P. Gong, Y. Osada, et al., Structural Characteristics of Double Network Gels with Extremely High Mechanical Strength, Macromolecules. 37 (2004) 5370-5374. doi:10.1021/ma049506i.

[18] L. Weng, A. Gouldstone, Y. Wu, W. Chen, Mechanically strong double network photocrosslinked hydrogels from N,N-dimethylacrylamide and glycidyl methacrylated hyaluronan, Biomaterials. 29 (2008) 2153-2163. doi:10.1016/j.biomaterials.2008.01.012.

[19] B. Trappmann, Extracellular-matrix tethering regulates stem-cell fate, Nat. Mater. 11 (2012) 642-649. doi:10.1038/nmat3339.

[20] M. Drigham, A. Bick, E. Lo, A. Bendali, J. Burdick, A. Khademhosseini. Mechanically robust and bioadhesive collagen and photocrosslinkable hyaluronic acid semi-interpenetrating networks. Tissue Eng. Part A. 15 (2009) 1645-1653. doi:10.1089/ten.tea.2008.0441.

[21] E. Dragan, M. Perju, M. Dinu. Preparation and characterization of IPN composite hydrogels based on polyacrylamide and chitosan and their 
inter0action with ionic dyes. Carbohydrate Polymers. 88 (2012) 270 281. doi:10.1016/j.carbpol.2011.12.002. 
Bis-acrylamide<smiles>C=CC(=O)NCNC(=O)C=C</smiles>

Acrylamide<smiles>C=CC(N)=O</smiles>

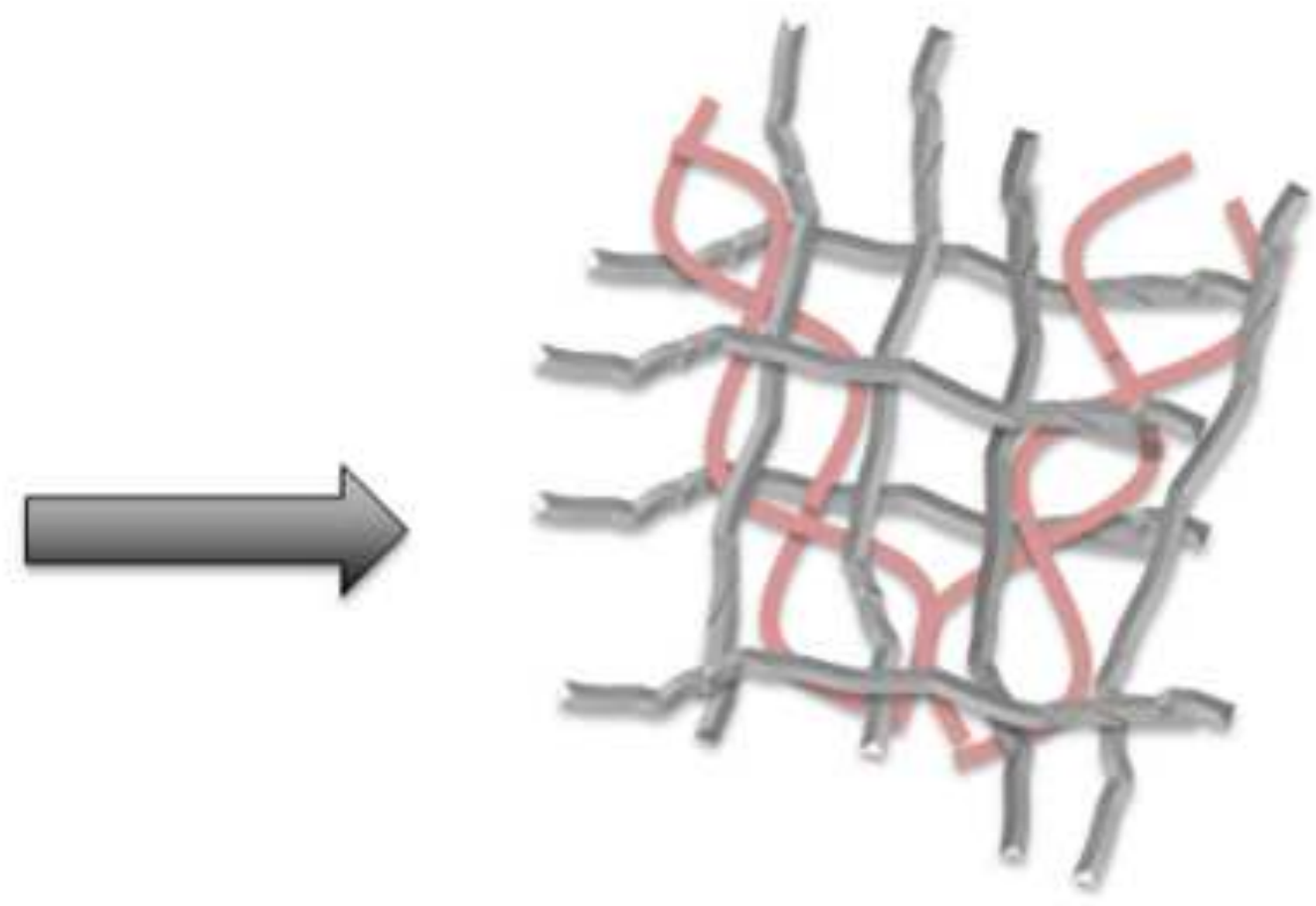

\title{
Collagen
} $\mathrm{NH}^{\mathrm{H}}$ $\mathrm{NH}_{2}$

\author{
PAAm

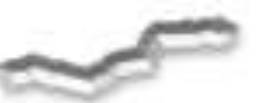 \\ Collagen
}

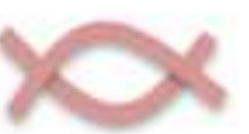

